\title{
eJRIEPS
}

Ejournal de la recherche sur l'intervention en éducation physique et sport

39 | 2016

Varia

\section{Une autochronie métaphorique constitutive de l'intelligibilité située des réalisations gymniques}

\section{Cathy Rolland}

\section{OpenEdition}

Journals

Édition électronique

URL : http://journals.openedition.org/ejrieps/795

DOI : 10.4000/ejrieps.795

ISSN : 2105-0821

Éditeur

ELLIADD

\section{Référence électronique}

Cathy Rolland, «Une autochronie métaphorique constitutive de l'intelligibilité située des réalisations gymniques », eJRIEPS [En ligne], 39 | 2016, mis en ligne le 01 juillet 2016, consulté le 01 août 2019. URL : http://journals.openedition.org/ejrieps/795 ; DOI : 10.4000/ejrieps.795

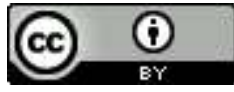

La revue eJRIEPS est mise à disposition selon les termes de la Creative Commons Attribution 4.0 International License. 
eJRIEPS 39 juillet 2016

Une autochronie métaphorique constitutive de l'intelligibilité située des réalisations gymniques

Cathy Rolland*

* Laboratoire ACTé (EA 4281), Université Blaise Pascal de Clermont-Ferrand, France.

Résumé

L'étude vise la caractérisation des processus cognitifs, largement implicites, que des entraîneurs experts en gymnastique artistique manifestent au cours de séquences d'enseignement des habiletés. Afin d'approcher les processus opératoires qui s'y expriment, l'activité qu'ils déploient au fil des interactions avec les gymnastes a fait l'objet d'une analyse selon une approche en anthropologie cognitive située. L'article met en relief la dynamique des processus cognitifs permettant aux entraîneurs de se rendre intelligible les tentatives de réalisation des gymnastes afin de les transformer. Les résultats montrent que les significations qu'ils élaborent en situation portent sur des unités discrètes successives, constitutives des habiletés. Ces unités révèlent des propriétés du mouvement qu'ils organisent en relations causales synchroniques et diachroniques. Cette attribution de sens se structure dynamiquement dans une temporalité générée par les entraîneurs, c'est-à-dire une autochronie.

Mots clés : Entraîneur - Intervention - Processus cognitifs - Autochronie -

"Accélère", "trop tôt", "plus vite"... Ces adresses verbales, intercalées entre les essais successifs des gymnastes sur les agrès, apparaissent familières aux habitués des salles d'entraînement. Relatives à l'organisation temporelle du mouvement, elles peuvent être considérées comme des interventions ordinaires pour des entraîneurs formés à la cinématique des gestes gymniques. Cette approche empirique, bien connue dans l'entraînement sportif, renvoie selon Perrey $(2007$, p. 204) à une analyse d'ordre qualitative dans la mesure où fondée sur l'observation in situ du mouvement, elle ne «permet pas de donner des valeurs précises de déplacement, de vitesse ou d'accélération du geste sportif réalisé, mais permet de définir une chronologie d'événements à l'origine de la performance ". Cette compétence à faire émerger du sens, 


\section{eJRIEPS 39 juillet 2016}

à partir d'un coup d'œil d'initié, s'élabore dans un contexte d'intervention dont l'originalité repose en partie sur la spécificité de l'activité gymnique. En effet, la pratique de la gymnastique artistique (GA) consiste en la réalisation d'enchaînements complexes d'une durée de 5 à 79 secondes selon les agrès (Jemni, Friemel, Sands \& Mikewski, 2001). Ces enchaînements combinent des éléments codifiés dont l'exécution varie de quelques fractions de secondes à quelques secondes et associe des déplacements linéaires et angulaires très rapides, combinés dans les trois plans de l'espace (Pozzo \& Studeny, 1987). Leur réalisation par les gymnastes requiert fondamentalement des qualités physiques de vitesse et de coordination (Salmela, 1982). La pratique de haut niveau, caractérisée par l'accentuation de la complexité des habiletés réalisées, exige ainsi une formation spécialisée dispensée par des professionnels expérimentés. Cette formation articule des périodes d'entraînement, structurées par des temps consacrés aux préparations physique, mentale et technique, et ponctuées par les tests de sélection et les épreuves compétitives. Ces temps sociaux instaurés par les centres de formation et les instances dirigeantes (Fédération Française et Internationale de Gymnastique Artistique) génèrent des contextes temporels d'intervention originaux, sources de problématiques pour les intervenants chargés des apprentissages. Une part importante du temps de formation est consacrée à la préparation dite technique (Fédération Française de Gymnastique (FFG), 1997, 2003) «qui vise à l'acquisition, à l'affinement, voire à l'invention, de savoir-faire répertoriés et considérés comme des formes optimales de réalisations motrices" (Durand, Hauw, Leblanc, Saury \& Sève, 2005, p. 56). Cette préparation repose sur une mise en pratique des gymnastes qui consiste à les faire s'exercer à la réalisation d'habiletés qu'ils ne savent pas encore faire. Ainsi, l'activité de régulation ${ }^{1}$ des apprentissages des gymnastes s'inscrit dans des horizons temporels conjoints : l'immédiateté des tentatives d'exécution des gymnastes et leur inscription dans une dynamique de transformations attendues à plus ou moins long terme (Durand, 2002). Elle révèle une compétence à se rendre intelligible les réalisations complexes des gymnastes dans des circonstances d'intervention originales. L'étude empirique présentée (Rolland, 2011) visait la caractérisation de ces activités d'optimisation des performances et la description des connaissances qui s'y expriment.

\footnotetext{
1 «On parle de régulation lorsque la reprise A' de l'action A est modifiée par les résultats et l'analyse de cette action A. » Durand (2002, p. 197)
} 


\section{eJRIEPS 39 juillet 2016}

\section{L’habileté gymnique comme phénomène de connaissance}

1.1. L'habileté gymnique comme mouvement reconstitué dans le temps de la physique $^{2}$

Le mouvement corporel est habituellement considéré comme déploiement spatiotemporel, changement de positions dans l'espace saisies dans une chronologie relative à une logique linéaire et quantitative du temps. Son analyse cinématique, descriptive et chronologique, s'est appuyée sur des dispositifs capables de conserver des traces de cet événement fugace pour l'observateur. Ainsi, la chronophotographie (Figure 1), développée au $19^{\text {ème }}$ siècle dans une logique scientifique et artistique, saisit des instantanés d'attitudes isolées et permet de reconstituer le mouvement au moyen de leur présentation en succession. Le zoopraxiscope, mis au point par Muybridge, projette ainsi une recomposition du mouvement au moyen d'un défilement cadencé des phases préalablement isolées.

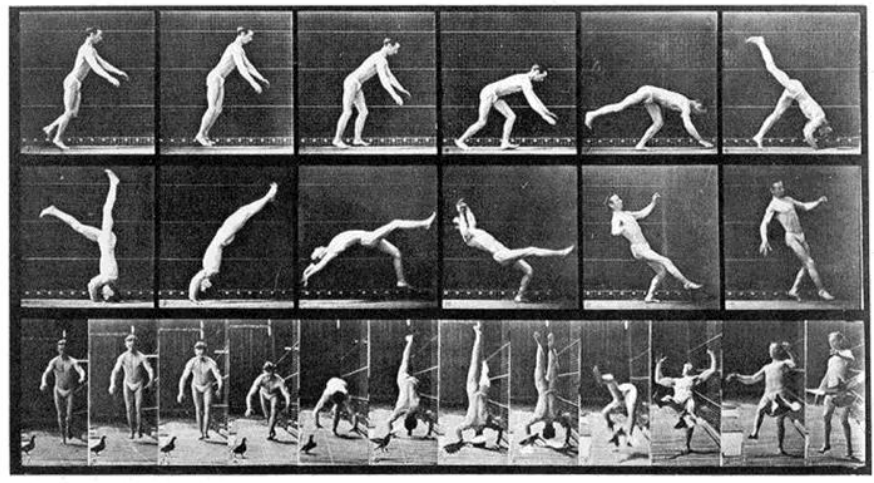

Figure 1. "Head-spring - A Flying Pigeon Interfering" (Muybridge, 1887).

Ces procédés de figuration du mouvement en deux dimensions ont été utilisés dans le domaine de l'entraînement sportif, particulièrement en gymnastique artistique dans la mesure où la finalité de l'activité sportive consiste en la reproduction de formes corporelles considérées comme justes par un groupe social (Goirand, 1986). Ainsi, en tant que produit attendu, ces formes ou habiletés motrices sont recensées dans des codes de référence (qui organisent le jugement des productions) et sont présentées dans des ouvrages de référence à destination des enseignants. Elles apparaissent sous forme de chronophotographies (Bourgeois, 1980 ; Kaneko, 1980 ; Koch, 1980 ; Mémento FFG, 1997) généralement accompagnées d'analyses dites descriptives et biomécaniques. Les

2 «La conception du temps en physique est subordonnée à la définition du mouvement ; de ce fait, le temps devrait être pensé comme un écoulement uniforme, identifié à l'espace et mesurable » (Rosenthal, 1993, p. 202) 


\section{eJRIEPS 39 juillet 2016}

descriptions formalisées des habiletés associent fréquemment chronophotographies, représentations imagées et verbalisations. Ces dernières détaillent chronologiquement pour chaque étape, considérée comme importante et présente parmi les photographies et/ou schémas, les positionnements corporels à adopter ainsi que les déplacements successifs et simultanés à effectuer afin de passer de l'un à l'autre. Ainsi, les représentations d'habiletés, sous forme de kinogrammes constitués de schémas juxtaposés (Figure 2), apparaissent relever d'un procédé de simplification représentationnelle conduisant à abstraire ce qui pourrait constituer leurs structures fondamentales.

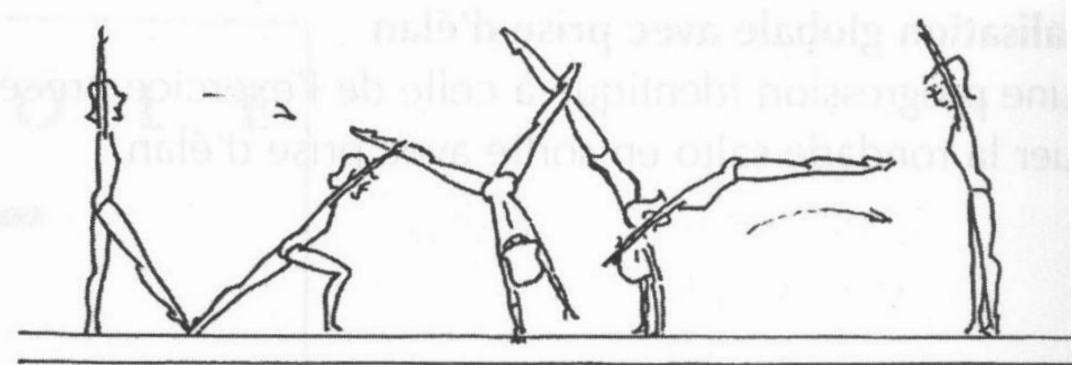

Figure 2. Représentations imagées de l'habileté gymnique rondade à l'agrès poutre (Mémento 1997 des activités gymniques).

Sous l'évidence de ce processus d'abstraction, communément mobilisé pour représenter les habiletés, apparaît l'opacité des éléments abstraits et ce qu'ils sont sensés révéler : ce qu'un observateur repère du mouvement ? Ce qu'un juge voit et évalue ? Ou encore ce qu'un entraîneur devrait faire apprendre ou ce qu'il enseigne effectivement ? Dans ce dernier cas, leur fonction passerait ainsi de représentation du produit attendu à celle de ressources pédagogiques permettant de guider les apprentissages des gymnastes.

\subsection{L'habileté gymnique comme objet d'enseignement/apprentissage déterminé} Cette dernière hypothèse conforterait le constat effectué par Nouillot \& Natta (2002) selon lequel « les enseignants et entraîneurs ne se contentent pas de donner à imiter une forme brute; ils choisissent dans cette forme des postures à des instants donnés qu'ils considèrent comme déterminantes pour la réussite des élèves ». Pour Kohler \& Nachon (2002), ces postures, ainsi que les rythmes du mouvement, font l'objet de démonstrations destinées à guider les gymnastes. Ces démonstrations, en tant que représentations 


\section{eJRIEPS 39 juillet 2016}

simplificatrices du geste à réaliser, caractérisent en partie, selon les auteurs, l'activité des enseignants expérimentés.

Constitutives des ressources formalisées destinées aux entraîneurs, ces descriptions cinématiques contribuent au développement d'une perspective objectivée du mouvement gymnique dont les conditions de production méritent d'être questionnées dans la mesure où elles entretiennent des liens, largement implicites, avec les pratiques d'intervention auprès des gymnastes. Ces formalisations professionnelles spécialisées légitiment les propositions de description cinématique en les associant à des considérations mécaniques, créant ainsi un modèle de rationalité épistémique valorisé dans les domaines de la formation des personnes (Perrenoud, Altet, Charlier \& Paquay, 1996) : l'argumentation scientifique. Fondées sur des raisons théoriques, atemporelles et dogmatiques (Koren, 2011), leur lien aux pratiques devient prescriptif, prédictif, mais aussi en partie descriptif, dans la mesure où ce qui fait l'objet d'un processus d'explicitation rationalisé sont des pratiques. Cependant, si les ouvrages pédagogiques entremêlent aux modèles académiques un patrimoine expérientiel incorporé, leur posture objectivante laisse dans l'ombre l'intelligence intuitive du corps, holistique et implicite, en prise avec le monde (Kaufmann, 1997).

Envisagé comme un praticien réflexif (Schön, 1983) qui élabore des solutions originales ajustées à la complexité des situations d'intervention, l'entraîneur génère des connaissances qui articulent des champs de savoir que les objectivations thématiques ont conduit à catégoriser et a priori penser séparément. Ainsi, la connaissance de la matière (Shulman, 1987) constitue selon Schneuwly (2012) une catégorie trop souvent non questionnée dans la mesure où sa détermination préalable en tant qu'objet de savoir à enseigner détourne la réflexion sur les procédures d'ajustement de sa transmission, sur la relation pédagogique. Les travaux de Shulman (1987) et Grossman (1990) ont permis de dépasser ce cloisonnement et d'envisager les «connaissances pédagogiques de la matière » (Pedagogical Content Knowledge ou PCK) comme l'articulation continue de la connaissance de la matière, des élèves, du contexte scolaire, de la pédagogie. Dans le domaine de l'entraînement sportif, la thématique de recherche nommée coaching a progressivement investigué les connaissances impliquées dans l'activité des praticiens, en particulier ceux qualifiés d'experts. Ces travaux ont permis d'éclairer ce que peuvent être les habiletés gymniques comme objet d'enseignement façonné par l'expérience de sa transmission. 


\section{eJRIEPS 39 juillet 2016}

1.3. L'habileté gymnique dans le temps expériencié de son enseignement

En rupture avec une conception algorithmique de la prise de décision, propre à la théorie computationnelle et considérée inadaptée pour expliquer l'activité réelle (Jones, Housner \& Kornspan, 1997 ; Tanguay \& Tousignant, 1998), les travaux de recherche s'attachent à comprendre les processus décisionnels des entraîneurs. Cette visée compréhensive, innovante dans un contexte scientifique marqué par les conceptions cognitivistes classiques, s'exprime sur les plans théorique et méthodologique. Les situations professionnelles ne sont plus réduites à des problèmes pré-déterminés et l'activité professionnelle à une capacité à se les représenter tels qu'ils sont normativement formalisés et prescrits. Ainsi, les connaissances sont envisagées comme des élaborations mentales fonctionnelles (Penelaud, 2010) dans la mesure où elles constituent des ressources permettant de résoudre des problèmes locaux. Leur mise à jour, nécessitant la considération du point de vue des acteurs, a mobilisé des techniques d'entretien innovantes (Caverni, 1988 ; Ericsson \& Simon, 1984 ; Hoc, 1984). Dans l'étude princeps de Salmela, Russel, Côté \& Baria (1994) et celle d'Irwin, Hanton \& Kerwin (2005), les entraîneurs d'élite en gymnastique artistique ont décrit verbalement leur activité en l'envisageant dans les situations variées et contrastées suggérées par les chercheurs. Les commentaires des entraîneurs portaient alors sur une activité susceptible d'être développée dans des circonstances dont l'originalité et la complexité étaient réduites dans les questions du chercheur à quelques caractéristiques (âge et niveau des gymnastes, moment d'intervention, etc.). Les catégories émergentes des procédures qualitatives et inductives de traitement des matériaux constituent pour les chercheurs une base de connaissances qui permet l'élaboration de modèles mentaux (Johnson-Laird, 1983). Ces modèles sont des configurations de connaissances dynamiquement élaborées, ajustées au problème singulier à résoudre. Bien que dépassant l'idée d'une activité cognitive comme mobilisation de connaissances mémorisées, les connaissances constitutives des modèles apparaissent comme des représentations amodales (Barsalou, 2008), redescriptions symboliques détachées de leur inscription sensori-motrice. Ainsi, ces travaux s'inscrivent encore dans une épistémologie de la représentation conçue comme processus relationnel entre un monde pré-donné, doté de propriétés extrinsèques et un sujet pourvu de symboles mentaux qu'il est sensé manipuler pour s'y adapter. En rupture avec cette conception dualiste, notre étude a adopté un présupposé pragmatiste (Steiner, 2008) considérant l'avènement contingent des propriétés du monde en lien avec les interactions pratiques de l'acteur avec sa situation. Ainsi, ce sont les propriétés 


\section{eJRIEPS 39 juillet 2016}

émergentes et signifiantes pour l'entraîneur (particulièrement celles relatives aux habiletés observées et corrigées), constitutives de la situation qu'il élabore en lien avec l'action exercée sur l'activité des gymnastes, qui caractérisent les connaissances comme phénomènes cognitifs expérienciés.

L'étude s'inscrit dans une approche qualifiée d'analyse de l'activité (Durand et al., 2005), qui vise, par l'adoption d'une posture compréhensive, à saisir l'expérience subjective des entraîneurs dans la dynamique de leurs interventions et la décrire sans s'enfermer dans les catégories usitées des points de vue formels, prescriptifs ou techniques. Elle ambitionnait la description des élaborations cognitives des entraîneurs experts dans la dynamique temporelle, expérientielle, de leur activité effective (en partie observable) d'optimisation des réalisations successives des gymnastes. Pour ce faire, elle a adopté une perspective théorique de la cognition qui réhabilite la corporéité d'une intelligence cognitive autonome en reconnaissant chez l'acteur une conscience, une expérience et un engagement dans le monde (Coste, 2003).

\section{Cadres théoriques et méthodologiques}

\subsection{Ancrages théoriques}

En rupture épistémologique avec le représentationnisme, le paradigme de l'enaction (Maturana \& Varela, 1994 ; Varela, 1989 ; Varela, Thompson \& Rosch, 1993) et les théories de la cognition situées (Greeno, 1998 ; Kirshner \& Whitson, 1997 ; Suchman, 1987) substituent au dualisme sujet/objet, l'idée de l'engendrement simultané du sujet et de son monde phénoménal. Le système cognitif est envisagé comme un processus relationnel, un couplage structurel asymétrique (Varela, 1989) dans la mesure où il provoque l'émergence de domaines cognitifs, zones de perturbations acceptables pour la préservation de la structure interne du système autopoïétique (Maturana \& Varela, 1994) qu'est l'acteur. Ces domaines propres à l'acteur, qui adviennent en même temps que le monde sur le mode de la question (Costes, 2003), s'expriment dans des actions situées et incarnées, un flux d'interactions privilégiées, « expression de son savoir-faire » (Rosenthal, 1993, p.200). Appréhender la dynamique de construction des perspectives signifiantes des entraîneurs suppose en conséquence de saisir leur ancrage dans le flux de leurs expériences d'interactions avec le monde (Barsalou, 2008). La caractérisation des habiletés comme processus cognitifs émergents, révélateurs d'une intelligibilité 


\section{eJRIEPS 39 juillet 2016}

pratique à «pénétrer un monde partagé ${ }^{3}$ » (Costes, 2003), implique de procéder à une lecture participative du système cognitif qui consiste à considérer le point de vue des entraîneurs sur leur propre activité (Varela et al., 1993).

\subsection{Procédures méthodologiques}

L'investigation a visé la saisie les processus cognitifs que les entraîneurs font émerger lors de séquences d'intervention au cours desquelles ils observent les réalisations d'habiletés pour les transformer, dans les conditions écologiques complexes de leur effectuation et de leur inscription dans une dynamique d'apprentissage.

\subsubsection{Participants et situations investiguées}

Les 14 entraîneurs experts qui ont collaboré à l'étude constituent une unité sociale cohérente dans la mesure où leur identité professionnelle commune et reconnue d'entraîneur ou éducateur en GA révèle une culture de l'entraînement partagée. Leurs pratiques d'intervention sont le produit de l'incorporation de savoirs professionnels, dispensés lors des formations initiales et continues, et co-construits lors de l'exercice du métier dans les salles d'entraînement, dans la mesure où l'usage spatial de celles-ci rend les pratiques d'autrui en partie visibles et audibles ${ }^{4}$, et favorisent les interactions. Les pratiques d'entraînement étudiées sont celles de professionnels désignés experts par leurs pairs et membres de la communauté gymnique. Leur notoriété repose notamment sur leur expérience passée de gymnastes de niveau national ou international, sur leur engagement professionnel de plus de dix années dans la gymnastique de performance ${ }^{5}$. En effet, deux d'entre eux exercent dans un club féminin de niveau national, alors que les douze autres ont été recrutés par deux Pôles France ${ }^{6}$ de la FFG afin d'assurer la formation des élites nationales. L'enquête de terrain s'est déroulée consécutivement sur les trois terrains sur des périodes d'au moins une année offrant l'occasion d'approcher les pratiques d'enseignement d'habiletés diverses, aux différents agrès (sol, poutre, barre fixe, etc.), pour des pratiquants de 11 à 23 ans et dans des dynamiques temporelles articulées: la séance, la semaine de formation et la saison sportive. Les entraîneurs (nommés dans le texte au moyen des lettres $\mathrm{A}$ à $\mathrm{N}$ ) ont la responsabilité de la formation

\footnotetext{
${ }^{3}$ Les significations ont une dimension sociale et culturelle dans la mesure où elles émergent dans un contexte socio-culturel original et qu'elles contribuent à son évolution (...Car la culture donne forme à l'esprit, Bruner, 1997).

${ }^{4}$ L'espace social, ouvert et libre, est un opérateur de normes selon Kaufmann (corps de femmes, regards d'hommes, sociologie des seins nus sur la plage, Kaufmann, 2010).

${ }^{5}$ La gymnastique de performance se différencie de la gymnastique de loisir par la finalité de sa pratique orientée vers la compétition à divers niveaux (régional, interrégional, national et international).

${ }^{6}$ Un Pôle est une structure d'entraînement labellisée par le Ministère de la Jeunesse et des Sports et de la Vie Associative et qui a pour but de former les sportifs au Haut Niveau tout en assurant leur suivi sportif et professionnel (FFG).
} 


\section{eJRIEPS 39 juillet 2016}

d'un groupe de gymnastes de niveau et âge similaires, sur des périodes annuelles ou pluriannuelles.

\subsubsection{Construction et analyse des matériaux}

La procédure méthodologique élaborée visait à documenter l'activité des entraîneurs, c'est-à-dire ses composantes manifestes (leurs comportements observables et descriptibles) et significatives. Les comportements ont fait l'objet de descriptions chronologiques détaillées et contextualisées à partir d'enregistrements audiovisuels et de notes ethnographiques. En tant que face visible d'une activité considérée, dans une posture compréhensive, comme fondamentalement énigmatique pour le chercheur, ils ont constitué le support d'entretiens destinés à faire expliciter (Vermersch, 1990) aux entraîneurs ce qu'ils avaient perçu dans les réalisations des gymnastes, leurs interprétations de ces aspects, signifiants pour eux, ainsi que les intentions de transformations sous-tendant leurs interventions auprès d'eux. Le dévoilement des propriétés phénoménales de leurs expériences a nécessité l'instauration progressive et négociée d'un dialogue asymétrique plaçant les protagonistes dans un rôle dynamique de construction d'un compromis de sens, favorable à la construction de matériaux en seconde personne (Vermersch, 1994). Ces interactions dialogiques, intercalées entre deux séquences d'intervention ou situées en fin d'entraînement, se sont concrétisées par des échanges au cours desquels l'entraîneur instruisait le chercheur (qu'il avait reconnu comme novice dans l'enseignement de certaines habiletés) sur sa propre activité.

L'analyse des matériaux, portant sur 104 séquences d'enseignement, visait l'élaboration d'un modèle théorique intégrant les éléments de généricité mis à jour à partir de l'approche qualitative et inductive de la grounded theory (Glaser et Strauss, 1967). Pour ce faire, les occurrences de signification, situées dans leur contexte d'émergence, ont fait l'objet d'une comparaison continue (Strauss, 1992) et exhaustive consistant à créer une catégorie conceptuelle (complétant les précédentes, ou amenant à les préciser) chaque fois qu'une occurrence offrait une nouvelle unité de signification.

\section{Résultats}

Les entraîneurs connaissent les habiletés qu'ils enseignent comme succession de phases transitoires. Cette figuration opérationnelle du mouvement qui fonde leur activité perceptive concilie des attentes de transformation conjointe de placements du corps dans l'espace et de déplacements dans le temps. Les entraîneurs interviennent sur les propriétés d'ordre physionomique et intentionnel qu'ils assignent à ces formes de corps, 


\section{eJRIEPS 39 juillet 2016}

dans la situation originale d'intervention. Ces formes constituent des totalités dont la signification repose sur un réseau de relations causales élaboré au fil des expériences de transmission.

3.1. Les phases de placement : des transitions spatio-temporelles ajustées Lorsqu'ils enseignent des habiletés, les entraîneurs experts centrent leur activité d'observation des réalisations ${ }^{7}$ et d'intervention auprès des gymnastes sur des portions de mouvement qu'ils nomment phases de placement (PP). Ces fragments constituent pour eux des jalons critiques qui ponctuent le mouvement comme autant de points névralgiques structurant leur activité conjointe avec les gymnastes. La fonction critique de ces phases repose pour eux sur deux caractéristiques phénoménales: a) une dimension «topo/morpho graphique » (Le Paven, 2008) du corps en mouvement à un instant précis de son déplacement, c'est-à-dire une forme de corps située dans l'espace d'évolution ; b) une dimension temporelle favorable à son effectuation passagère, intercalée entre ses phases antérieure et postérieure. Leur exécution vise à assurer une mise en trajectoire optimale vers la séquence transitoire suivante, ce qui suppose une articulation fine sur les plans conjoints d'espace et de temps avec les phases qui les encadrent. La description que l'entraîneur D adresse au gymnaste associe des défectuosités relatives à l'organisation temporelle (une réalisation de la phase écourtée) à des défectuosités liées à l'organisation spatiale du mouvement (un placement corporel non abouti) : «Tu vas trop tôt ; tu n'as pas suffisamment de placement ».

Les PP constituent pour les entraîneurs des étapes réorganisatrices des réalisations spontanées des gymnastes au cours desquelles ils présentent une tendance à négliger certaines séquences que les entraîneurs jugent capitales ou écourtent leur réalisation, n'atteignant pas ainsi le placement corporel favorable aux déplacements ultérieurs. L'enseignement dispensé consiste alors à focaliser les gymnastes sur ces phases transitoires, plus précisément à prolonger dans le temps leur effectuation. C'est précisément l'intention de l'entraîneur I concernant la réalisation de la phase transitoire de balancé avant (Photographies 3 et 4) précédant une sortie en salto arrière tendu à la barre fixe. II fait répéter les balancés avant et arrière et commente ensuite leur qualité au gymnaste tout en lui faisant visionner l'enregistrement audiovisuel de sa prestation: «Bon, tu sais faire courbe arrière et avant ; regarde. » L'entraîneur effectue un arrêt sur

\footnotetext{
${ }^{7}$ Les expressions indigènes mobilisées par les entraîneurs pour qualifier l'objet de leur attention ont été conservées. Le « mouvement », la « réalisation » désignent ainsi l'action motrice en tant qu'événement phénoménal pour les entraîneurs, sans présager des significations que ces événements acquièrent pour eux en cours d'intervention.
} 
image sur la phase de balancé avant (Photographie 3) : "Tu as vu ? Si tu lâches làdessus (Photographie 4), tu fais double arrière en sortie ».

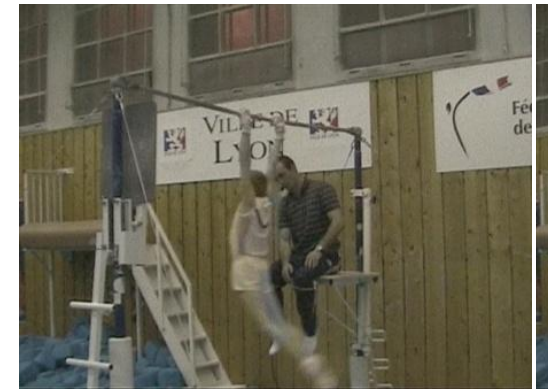

Photographie 1.

Passage en courbe arrière.

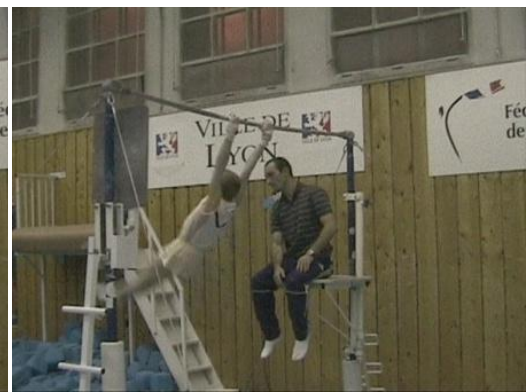

Photographie 2.

Amorce du balancé avant.

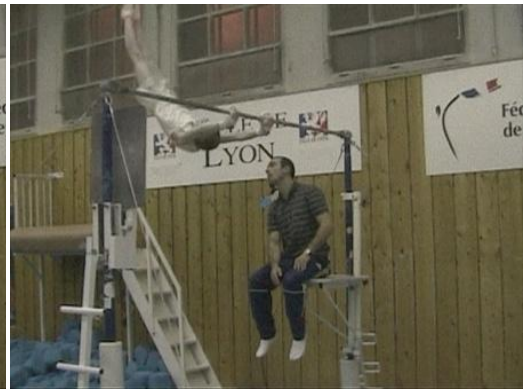

Photographie 3.

Courbe avant.

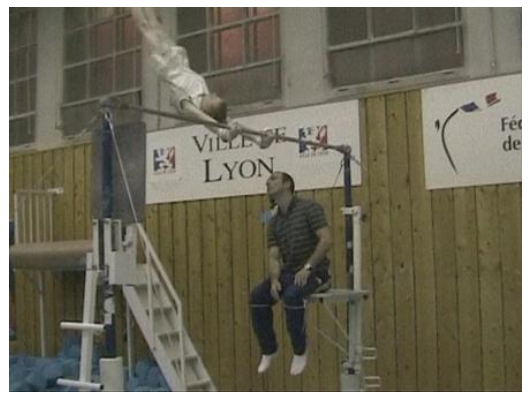

Photographie 4. Fin du balancé avant.

Or le gymnaste ne réalise pas cette courbe au moment où il lâche l'agrès (photographie 5) pour effectuer le salto arrière (Photographie 6). L'entraîneur L, qui co-intervient sur cette séquence avec l'entraîneur I, décrit au chercheur ce qu'il perçoit et ses préoccupations afférentes : " on voulait qu'il sorte en passant par cette position en courbe avant, le centre de gravité à peu près au niveau de la barre, le regard sur les pointes de pieds, parce que je ne sais pas si tu as vu mais $D$. quand il fait sa sortie, il tire la tête et après, ça lui fait sortir la poitrine ».

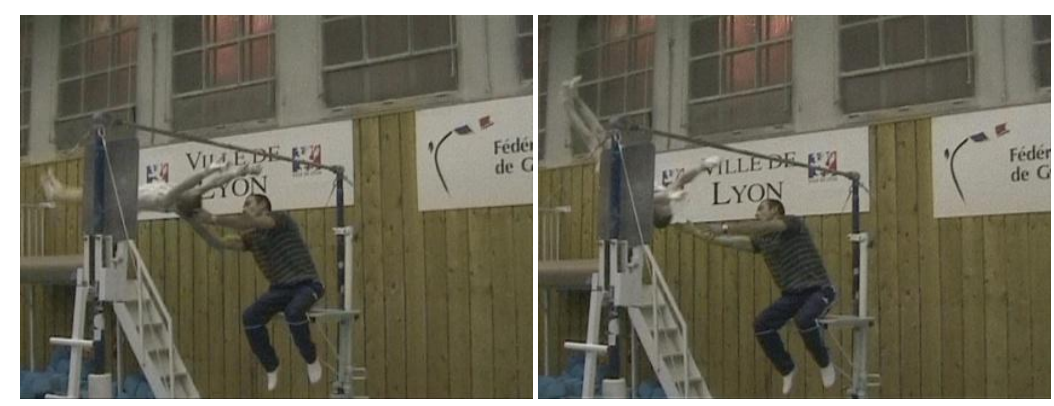

Photographies 5 et 6 


\section{eJRIEPS 39 juillet 2016}

L'entraîneur I manipule alors le gymnaste afin de le placer précisément dans la position attendue. Quant à l'entraîneur L, il aménage l'environnement de pratique en y ajoutant un bloc de mousse comme cible à atteindre avec les pieds à la fois dans les balancés avant préparatoires (Photographie 7) et au moment du lâcher (Photographie 8) : « Tu vois quand tu lâches la barre, les pointes de pied ne touchent même pas la mousse. Tu imagines à quelle hauteur tu es ?! ».

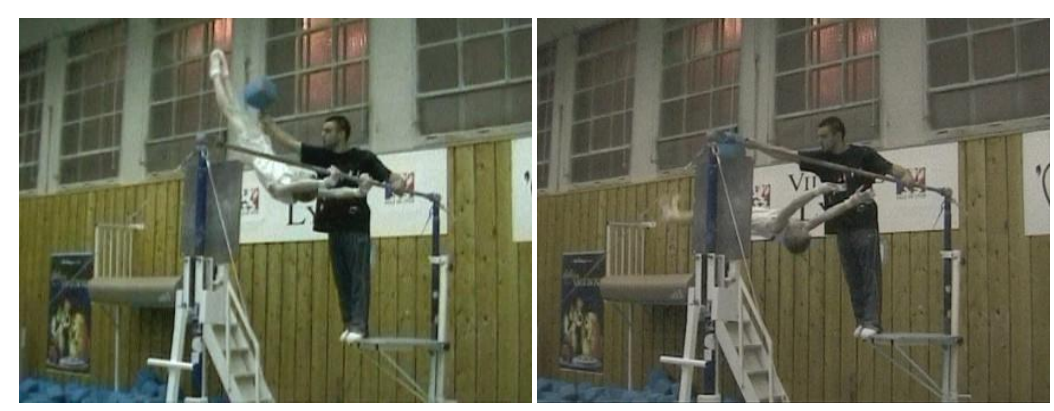

Photographies 7 et 8

Des formes d'intervention différentes et complémentaires, destinées à améliorer les PP, sont également mobilisées. Les entraîneurs effectuent des descriptions verbales ou graphiques aux gymnastes en dessinant à la craie sur un tableau ou à la magnésie sur un tapis les formes corporelles situées, caractéristiques de la phase ; ils représentent aussi ces formes en les mimant avec leur corps ou en usant de leurs doigts et avant bras (Photographies 9 et 10). Ces représentations mettent en évidence l'habileté comme flux de formes corporelles situées dans l'espace et masquent une problématique inhérente à leur enseignement : les régulations destinées à faire prolonger leur réalisation nuisent à la dynamique du mouvement et à son accomplissement jusqu'à son terme : «Tu as essayé mais ça retombe, parce que tu restes trop longtemps ; c'est dans la continuité : j'enroule et je tourne » (Entraîneur D).

L'ajustement fonctionnel des PP comme placements transitoires assurant le déploiement d'un mouvement fluide, équilibré, ample et composé de rotations terminées, suppose ainsi une régulation optimale de l'activité du gymnaste qui s'opère in situ, au moyen de processus cognitifs complexes.

3.2. La cohérence pragmatique des phases de placement

3.2.1. Une cohérence synchronique

La PP possède pour les entraîneurs une cohérence physionomique qui intègre des propriétés de différents ordres (géométrique, topologique, micro-temporelle et dynamique), 


\section{eJRIEPS 39 juillet 2016}

organisées en système par un réseau de liens logiques causaux. Cette connaissance systémique, enrichie au fil des tentatives d'optimisation des réalisations, offre aux entraîneurs des voies de régulation diversifiées pour faire face à l'originalité des situations d'intervention. Lorsque l'entraîneur I indique au gymnaste son erreur de placement de la tête à la fin de la phase de balancé avant (Photographie 9), il intervient sur une dimension géométrique qu'il assigne à la phase et qui caractérise l'espace postural en termes de types géométriques (lignes (droit, tendu, fermé, plié), courbes (creux, en extension), angles (ouvert ou fermé)). Quant à lui, l'entraîneur $L$ tente de modifier la réalisation en intervenant sur l'organisation topologique, c'est-à-dire la situation de la forme corporelle relativement à des référentiels de localisation spatiale émergents en situation (la barre ou la mousse par exemple) selon le placement adopté pour observer le mouvement. Tous deux cherchent la ficelle, le levier ou le ressort (selon l'image mobilisée pour représenter les éléments constitutifs de ce système causal) susceptible de provoquer une réorganisation globale des propriétés caractéristiques de la phase. Ressource implicite à l'émergence spontanée de propriétés nouvelles («ça m'est venu comme ça » Entraîneur C concernant une prescription innovante relativement aux précédentes), ce système constitue aussi un outil réflexif pour l'élaboration de nouvelles hypothèses explicatives des manques constatés.

\section{Extrait d'entretien avec l'entraîneur $L$ suite à la séquence de co-intervention avec l'entraîneur I orientée vers la transformation de la sortie en salto arrière tendu :}

EL : « Je ne crois pas qu'il puisse faire la sortie correctement en prenant cette position parce qu'il tire sur la barre. Il fait une action de bas en haut avec les bras au lieu de rester épaules basses. ”

$\mathrm{CH}:$ « Et comment tu l'as perçu? »

EL : « Je l'ai vu parce qu'il reste accroché à la barre. C'est le temps, il y a un temps trop long où il reste accroché. D’ailleurs, c'était I qui était obligé de le tirer un peu pour qu'il lâche. Donc j'ai pensé que quand il décide de lâcher la barre, il fait cette action avec les bras au lieu de rester épaules basses. C'est cohérent avec ce qu'il fait quand il lâche tout seul. ”

L'opération motrice "tirer sur la barre" (propriété dynamique) s'agrège à celle de "avoir la tête en extension et la poitrine sortie" (propriété géométrique), "lâcher trop tard" (propriété micro-temporelle), "avoir les pieds à l'horizontale" (propriété topologique). L'ensemble de 


\section{eJRIEPS 39 juillet 2016}

ces propriétés fournit à la PP sa physionomie, matérialisée par une forme de corps dynamique. L'entraîneur s'attache à la transformer lorsqu'il s'engage dans la situation d'intervention comme observateur d'un dé-placement (ou coordination de placements). Lorsque cet engagement spontané s'avère improductif pour générer les transformations attendues, un nouvel engagement s'y substitue, associé à l'émergence de propriétés complémentaires que nous qualifions d'intentionnelles dans la mesure où elles caractérisent ce que l'entraîneur saisit du contrôle intentionnel du mouvement par le gymnaste. Ce sont des propriétés d'intention praxique (ce que le gymnaste doit chercher à faire), d'intention de perception visuelle et de sensations (ce que le gymnaste doit chercher à voir et à percevoir en cours d'action), d'intention d'attention (ce à quoi le gymnaste doit prêter attention pendant qu'il agit).

Le lien que l'entraîneur $L$ établit progressivement entre le mouvement constaté de la tête et les intentions praxiques du gymnaste lui offre un changement de prises sur l'activité du gymnaste : "ça fait déjà quelque temps qu'on réfléchit avec I. On a choisi ensemble de travailler sur la posture au moment du lâcher mais quand on voit que ça ne marche pas, il faut savoir changer de direction. Là, je pense qu'il faut revenir à la sortie. Je pense qu'il a peur et qu'il ne peut pas chercher à faire cette courbe ».

Ainsi la position de la tête « en extension » au moment du lâcher peut être corrigée en décrivant au gymnaste ce qu'il donne à voir (Photographie 9) ou encore en recommandant au gymnaste « soit de chercher à venir mettre le menton à la poitrine, soit de chercher à sentir le menton à la poitrine ou à voir le nombril » (Entraîneur G), soit de « voir ses pointes de pieds » (Entraîneur I). Ces opportunités de guidage des apprentissages suppose de l'entraîneur un déplacement de point de vue, de celui d'observateur du mouvement à celui, plus empathique et délicat, du gymnaste sensé conduire et contrôler intentionnellement son déplacement.

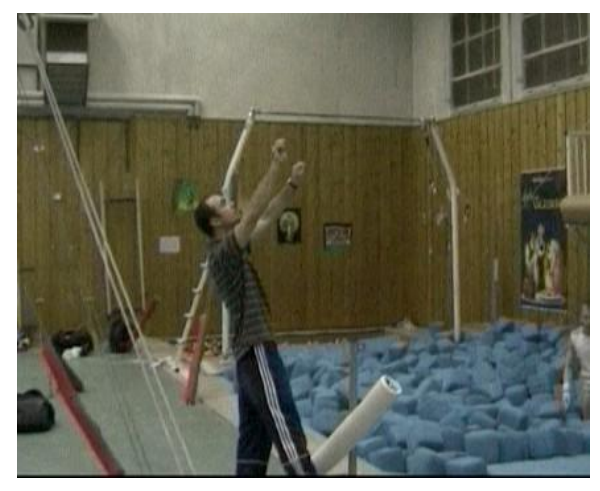

Photographie 9. L'entraîneur I demande au gymnaste « Tu vois tes pointes avec la tête comme ça ? », en mimant la position de la tête. 


\section{eJRIEPS 39 juillet 2016}

La connaissance que les entraîneurs développent des PP apparaît foisonnante. Leur description analytique, mettant à jour des propriétés de nature différente, révèle la richesse des perspectives descriptives et opératoires que les entraîneurs s'offrent pour guider les gymnastes. Pour autant, elle masque en partie leur expression émergente et la finesse avec laquelle elles s'ajustent aux circonstances originales d'intervention, telles que l'histoire du gymnaste (la qualité de ses apprentissages antérieurs), ses ressources (souplesse, puissance, etc.), le temps jugé disponible pour générer des transformations par rapport aux échéances à venir (compétitions). En effet, le système de relations causales synchroniques n'a pas une fonction déterministe. Les propriétés sont qualifiées au sein de la réalisation singulière du gymnaste. C'est, par exemple, l'interprétation que fait l'entraîneur $L$ de la réalisation du gymnaste qui qualifie la propriété micro-temporelle de la PP en balancé avant par un empan temporel exagérément prolongé (« lâcher trop tard»), associé à une action inappropriée des bras sur la barre ("tirer sur la barre»). Sans cette action sur la barre, l'empan temporel aurait pu être qualifié d'insuffisant car ne permettant pas d'amener le bassin à hauteur de la barre. Les propriétés émergent ainsi dans l'originalité de la production présente du gymnaste, insérée dans la dynamique de ses essais successifs.

\subsubsection{Une cohérence diachronique}

Les interventions régulatrices des entraîneurs sont ciselées au fil des interactions de travail avec les gymnastes. Les propriétés qu'ils assignent à une PP émergent d'une interprétation l'impliquant dans une chaîne causale historique, sa qualité ayant des implications sur celle de la phase postérieure. Les PP apparaissent comme des séquences chrono-logiques, la logique temporelle consistant en une cohérence diachronique structurée par, et structurante de formes dynamiques comme totalités signifiantes. L'ordonnancement logique diachronique que les entraîneurs confèrent aux PP soutient leur activité d'observation des réalisations (selon une suite séquentielle logique), ainsi que leur activité d'enquête lorsqu'ils n'identifient pas spontanément la séquence à optimiser.

Les propriétés topologiques de la PP (Dessin 2 de la Figure 3) que l'entraîneur D s'attache à améliorer, pour optimiser la réalisation d'un saut 1 tour $1 / 2$ au sol à l'appui facial (Figure 3 ), se constituent in situ en fonction des significations qu'il attribue aux phases postérieures: "être plus vertical" lorsqu'il repère une arrivée buste en avant; "fixer la trajectoire" lorsqu'il repère une arrivée décalée par rapport à une projection horizontale sur le tapis de l'axe vertical du corps. 


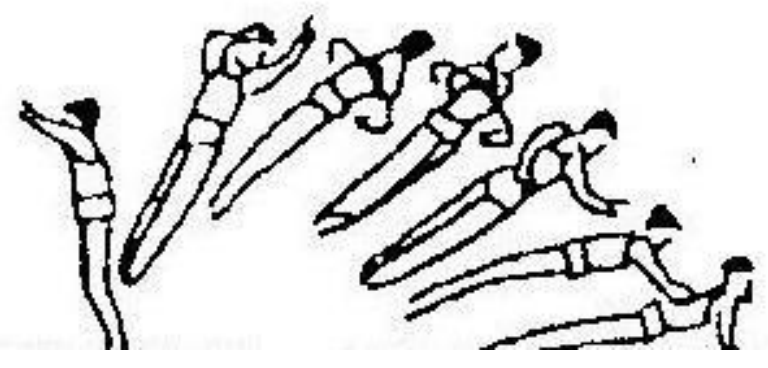

Figure 3. Représentation schématisée du saut 1 tour 1/2 au sol (Code FIG 1997).

Extrait d'interventions verbales adressées par l'entraîneur $D$ au gymnaste lors de la séquence d'enseignement du saut 1 tour $1 / 2$ à l'appui facial :

Après l'essai 9: "Tu es trop bas ; plus tu vas être vertical (il montre avec sa main légèrement inclinée (Photographie 10), plus c'est facile parce que c'est tes pieds qui vont toucher en premier ; linverse, c'est les mains".

Après l'essai 10: "Tu as vu comme tu désaxes ? Parce que tu ne fixes pas la trajectoire».

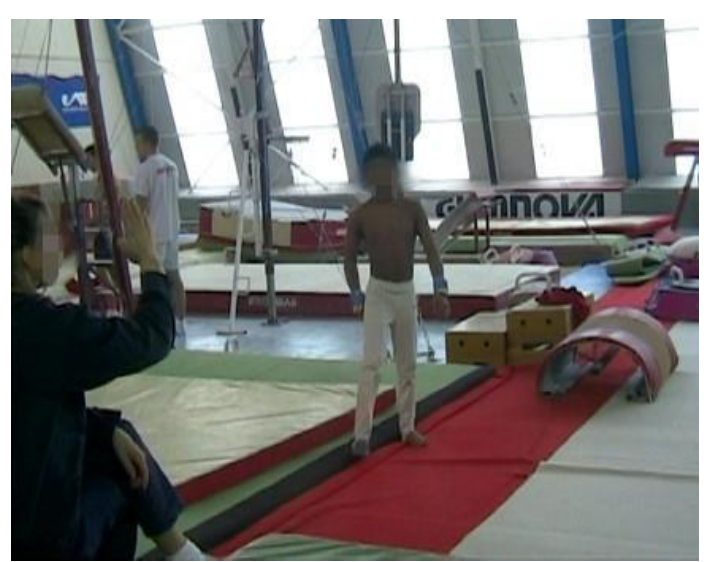

Photographie 10. L'entraîneur D mime avec sa main la phase de mise en trajectoire.

Au cours de cette séquence d'intervention, l'entraîneur $D$ insiste auprès du gymnaste sur la première phase du saut qu'il nomme « mise en trajectoire » du corps (Photographie 11). 


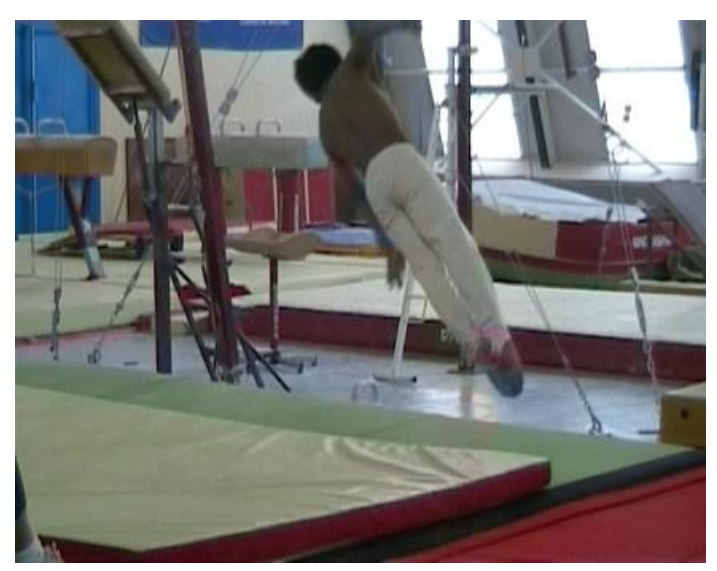

Photographie 11. Phase de mise en trajectoire lors du saut 1 tour $1 / 2$ à l'appui facial.

Ses interventions visent à prolonger la réalisation de cette phase (fixer la trajectoire) mais en avançant le moment de déclenchement de la rotation longitudinale afin que le gymnaste puisse voir le tapis : "Si tu restes longtemps là (il montre regard devant soi dans la phase d'impulsion et de mise en trajectoire), tu ne vas pas arriver à voir. " Les explicitations de l'entraîneur adressées au chercheur précisent le sens de ses interventions : "S'il ne se met pas en trajectoire il ne verra rien du tout et $A$. ce qu'il faisait c'est qu'il fixait longtemps devant et en fait sur tout ce qui est 1 tour $1 / 2$, je voulais qu'il regarde deux fois son tapis. "Cependant lorsqu'il incite le gymnaste à regarder le tapis situé derrière lui, l'entraîneur fait le constat d'un engagement trop rapide dans la rotation et de l'adoption d'un positionnement spatial du corps trop à horizontale le conduisant à nuancer sa demande : «En fait, faut pas leur demander d'aller voir non plus parce que sinon ils vont anticiper et ils ne vont pas rester sur la trajectoire. Mais l'idéal serait qu'il voit une première fois avant de faire le tour et de voir une deuxième fois ; il faut jouer sur ça... faut pas leur demander d'aller voir ".

La durée optimale de la phase de mise en trajectoire du corps se situe pour l'entraîneur D entre des exigences de perception visuelle (voir le tapis après le $1 / 2$ tour) et des exigences d'organisation topologique: adopter une trajectoire verticale incompatible avec une intention du gymnaste de regarder le tapis, intention propice à une anticipation de la rotation. La connaissance empirique de cette durée optimale émerge au fil des interventions successives de l'entraîneur qui oscillent entre ces deux exigences qu'il résume par l'engagement intentionnel vers lequel il pense pertinent d'orienter le gymnaste et qu'il résume ainsi au chercheur : " voir sans chercher à aller regarder le tapis ». 


\section{eJRIEPS 39 juillet 2016}

Extrait d'interventions verbales adressées par l'entraîneur D au gymnaste lors de la séquence d'enseignement du saut 1 tour $1 / 2$ à l'appui facial :

Après l'essai 1 (1/2 tour) : «Tu ne penses qu'à tourner ; refais en fixant la trajectoire. 》

Après l'essai 2 (1/2 tour) : "Si tu fais ça, le tour et demi est fait; si tu ne fais pas ça, il n'est pas fait. 》

Après l'essai 3 (1 tour $1 / 2$ ) : «Ça c'est mieux ; le problème c'est que tu ne regardes pas ton tapis sur le $1 / 2$ tour et donc après tu ne le vois pas sur l'arrivée ; refais la préparation en voyant le tapis.»

Après l'essai 4 (1/2 tour) : «Là tu tardes un peu pour venir voir ton tapis. 》

Après l'essai 5 ( $1 / 2$ tour) : «Voilà mais là tu es un peu trop à l'horizontale. Les repères en tour $1 / 2$, c'est là (il montre en tournant la tête derrière pour mimer $1 / 2$ tour) et là (il mime 1 tour et $1 / 2$ ); si tu restes longtemps là (il montre regard devant soi dans la phase d'impulsion), tu ne vas pas arriver à voir. ”

Après l'essai 6 (1 tour $1 / 2)$ : « Un peu mieux et encore, c'était un peu tôt; tu sens la différence ? Refais la préparation. »

Après l'essai 7 ( $1 \frac{1}{2}$ tour) : «Voilà ; il faut vraiment se concentrer sur ça, surtout pas sur la rotation. 》

Après l'essai 8 ( 1 tour $1 / 2)$ : «Non, tu as regardé devant. 》

Les interventions régulatrices s'ajustent au fil de l'interprétation de leur efficacité à générer des transformations au fil des essais des gymnastes. Leur affinement consiste à alterner des exigences parcellaires, parfois contradictoires, à les nuancer, à les combiner au sein de formules lexicales originales.

\section{Discussion}

4.1. La phase de placement : un oxymoron signifiant

L'idiome professionnel "Phase de placement " rend compte d'une catégorie mentale opératoire qui permet aux entraîneurs de se rendre intelligible les réalisations gymniques dans l'intention de les optimiser. Elaborée au fil des interactions dialogiques avec les gymnastes, la PP apparaît comme une « unité d'action » (Durand, 2002, p. 184) organisatrice des activités conjointes et articulées d'enseignement/apprentissage des protagonistes. Au sein de ces interactions, l'habileté est conçue comme l'agencement successif et coordonné de ces unités d'action, représentées et perceptivement identifiées comme des formes corporelles dynamiques discrètes. La PP, interne au mouvement, 


\section{eJRIEPS 39 juillet 2016}

détermine intentionnellement et physiquement la forme de ce dernier : il s'agit d'une «immobilité du mouvement», oxymoron signifiant. Cet alliage entre deux catégories sémantiques contradictoires révèle une tension cognitive et pragmatique : structurer le mouvement par des étapes spatiales précises tout en assurant la dynamique du déplacement. La PP est l'expression sémantique d'une intelligibilité pratique qui combine discret et continu, stabilité et dynamisme, forme repérable dans la continuité du mouvement pour l'entraîneur, forme à prolonger et à coordonner aux autres pour le gymnaste. Elle dévoile une perception phénoménologique qui dépasse une conception gestaltiste dans l'incapacité de régler cette tension inhérente à la nécessité pour les entraîneurs d'agir pour modifier le mouvement : soit le mouvement est perdu au profit de formes statiques, soit les formes sont perdues au profit du mouvement global (Gely, 2005). Non pas statiques, les formes corporelles gymniques sont des formes en tension en tant qu'elles constituent des raccords fictifs entre des éléments partiels d'un mouvement, et qu'elles permettent aux entraîneurs la perception du mouvement.

Sa discrétisation en formes corporelles dynamiques inscrit la signification du mouvement comme celle du monde sonore dans une ontologie d'événements (Molino, 1998) ${ }^{8}$. Elle apparaît comme un artefact dans la mesure où le phénomène événementiel « garantit l'identité qui résiste à la décomposition du temps », et constitue un repère métonymique organisateur des activités de l'entraîneur et du gymnaste. La forme « est le plus solide bouclier inventé par l'intelligence contre la fuite du temps» (Darbellay, 1998, p.16). Cependant, l'intelligibilité développée en situation par les entraîneurs ne confère pas à la forme des propriétés stables et préalablement fixées. L'équilibre dynamique qui intègre les formes corporelles au sein d'un continuum est lui-même un équilibre situé, ajusté à l'idiosyncrasie de la réalisation actuelle.

\subsection{L'épaisseur d'une microgenèse fondée sur un modèle d'intelligibilité}

Telle que perçue par l'entraîneur, la PP est structurée dans l'épaisseur d'une microgenèse du sens de ce mouvement (Rosenthal \& Visetti, 1999). En tant qu'objet d'expérience, elle émerge d'un processus de déploiement progressif mais immédiat du sens (associé à un percept, une expression sémantique) qui consiste en un parcours catégoriel du général et indéfini vers le spécifique et défini. Pour les auteurs, le processus s'inscrit dans un Présent épais qui d'un côté retient la participation du passé et de l'autre, ouvre sur le futur

\footnotetext{
${ }^{8}$ Pour Molino (1998), c'est l'écoulement du phénomène sonore dans un continuum linéaire qui en permet la discrétisation, contrairement à la discrétisation du visuel (qui s'inscrit dans une ontologie d'objets stables) qui consiste en la construction des objets du monde, pour lesquels existe un nom qui garantit son identité et son autonomie.
} 


\section{eJRIEPS 39 juillet 2016}

immédiat. La microgenèse instancie ainsi un processus dynamique d'anticipation enactive de ce qui fera sens. Dans cette perspective phénoménologique, l'émergence du sens n'est pas compatible avec le concept physique du temps. Elle suppose une temporalité endogène, créée en propre par l'acteur qui potentialise son autopoïèse (Rosenthal, 1993). En tant que phénomène distinct et inscrit dans une continuité expérientielle causale, la PP témoigne d'une temporalité discrète, d'un découpage événementiel rendu possible par un principe d'autochronie (op. cit., 1993), d'autogénération du temps propre au sujet.

Ce parcours catégoriel consiste en l'enaction de certaines propriétés d'ordre physionomique ou intentionnel selon leur pertinence pragmatique pour agir dans la situation. La structuration de ces propriétés, dont le sens se spécifie dans le déploiement

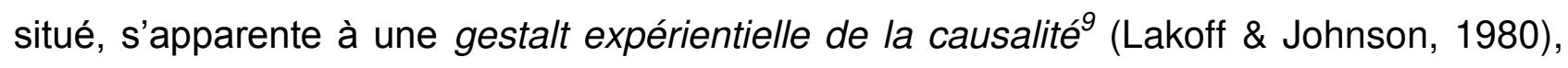
générée par analogie à une structure d'intelligibilité préalable des interactions corporelles à l'environnement physique. En effet, l'analyse des expériences, que les entraîneurs ont explicitées, révèlent une connaissance biomécanique «naïve ( Vosnadiou \& Brewer, 1992) nourrie de régularités empiriques constatées au fil des activités conjointes d'observation des mouvements et de guidage des apprentissages. Cette aire originelle d'intelligibilité, ou métaphore radicale (root - metaphors) (Pepper, 1942), structure in situ les expériences des habiletés gymniques en termes de dé-placement ou dé-placement intentionnellement conduit, et génère l'émergence associée d'une propriété opératoire d'ordre physionomique ou intentionnel. L'événement distinct dans l'habileté qu'est la $P P$ (intentionnellement conduite) constitutive d'un dé-placement (intentionnellement conduit) ${ }^{10}$ constitue une description analogique originale, permettant de répondre aux exigences de la situation d'intervention auprès des gymnastes.

\section{Conclusion}

L'habileté gymnique est saisie par les entraîneurs experts dans une autochronie qui autorise son déploiement comme processus dynamique, structuré par des phénomènes séquentiels distincts : les PP. La connaissance de ces phases, irréductible à une identité distincte, autonome et fixée telle que leur juxtaposition schématique dans les ouvrages de

\footnotetext{
${ }^{9}$ Structure multidimensionnelle qui 1) organise les expériences en ensembles cohérents ; 2) est organisée autour d'un noyau prototypique (Rosch, 1977) de la causalité dont l'émergence repose sur les expériences corporelles qui se spécifient métaphoriquement en empruntant par analogie une structure offrant des corrélations systématiques pertinentes pour l'acteur.

${ }^{10}$ L'usage des parenthèses souligne l'idée que les propriétés, associées à l'interprétation de la réalisation en termes de conduite d'un dé-placement, sont momentanément placées en retrait, dans l'ombre, car non exprimées dans la situation actuelle construite par l'acteur.
} 


\section{eJRIEPS 39 juillet 2016}

référence le laisse supposer, relève d'un développement situé de significations fondées sur des liens logiques entre des phénomènes parcellaires du mouvement. La mise à jour de cette intelligibilité située plaide en faveur de la conception de dispositifs de formation des entraîneurs «centré activité » (Bourgeois \& Durand, 2012), émancipée d'une épistémologie de la science appliquée dans l'incapacité de prescrire une activité d'ajustements continus à des circonstances complexes. S'agissant en conséquence d'accompagner favorablement le développement de l'activité professionnelle des entraîneurs novices selon des modalités réflexives, ces dispositifs (tutorat, vidéoformation par exemple) devraient prendre appui sur une analyse des processus situés d'élaboration de ce modèle d'intelligibilité à l'œuvre chez les experts (ce qui supposerait une étude complémentaire).

\section{Bibliographie}

Barsalou, L. (2008). Grounded cognition. Annual Review of Psychology, vol. 59, 617-645.

Bourgeois, M. (1980). Gymnastique sportive: perspectives pédagogiques école-club. Paris : Vigot.

Bourgeois, E., \& Durand, M. (2012). Apprendre au travail. Paris : PUF.

Briere-Guenoun, F., Perez, S., \& Durey, A. (2007). Etude exploratoire des connaissances mécaniques mobilisées par les enseignants d'EPS. Les conceptions des conseillers pédagogiques. Science et Motricité, 61/2, 9-23.

Bruner, J.S. (1991). Car la culture donne forme à l'esprit. Paris : Eschel.

Caverni, J.-P. (1988). La verbalisation comme source d'observables pour l'étude du fonctionnement cognitif. In J. Caverni, C. Bastien, P. Mendelsohn \& G. Tiberghien (Eds.), Psychologie cognitive : Modèles et méthodes (pp. 253-273). Grenoble : Presses Universitaires de Grenoble.

Coste, F. (2003). Incarnation, cognition et représentation : comment les sciences cognitives pensent-elles le corps ?, Tracés. Revue de Sciences humaines, 2. En ligne http://traces.revues.org/4135

Darbellay, E. (Ed.). (1998). Le temps et la forme. Pour une épistémologie de la connaissance musicale. Genève : Librairie Droz.

Durand, M. (2002). Chronomètre et survêtement. Reflet de l'expérience quotidienne d'enseignants en éducation physique. Paris : Editions Revue EPS. 


\section{eJRIEPS 39 juillet 2016}

Durand, M., Hauw, D., Leblanc, S., Saury, J., \& Sève, C. (2005). Analyse de l'activité et entraînement en sport de haut niveau. Education Permanente, 161, 54-68.

Ericsson, K. A., \& Simon, H. A. (1984). Protocol Analysis: verbal reports as data. Cambrige: MIT Press.

Fédération Française de Gymnastique. (1997). Mémento 1997 des activités gymniques. Toulouse : Cota Production.

Fédération Française de Gymnastique (2003). Mémento des activités gymniques. Toulouse : Cota Production.

Gely, R. (2005). Les usages de la perception. Réflexions merleau-pontiennes. Louvain-LaNeuve : Editions de l'institut supérieur de philosophie \& Louvain, Paris : Editions Peeters.

Glaser, B.G., \& Strauss, A.L. (1967). The discovery of grounded theory: Strategies for qualitative research. Chicago: Aldine.

Goirand, P. (1986). Apprendre en EPS en faisant de la gymnastique. Spirales, 1, 11-46.

Greeno, J.G. (1998). The situativity of knowing, learning and research. American Psychologist, 53, (1), 5-26.

Grossman, P.L. (1990). The making of a teacher: Teacher knowledge and teacher education. New-York: Teacher College Press.

Hoc, J.-M. (1984). La verbalisation provoquée pour l'étude du fonctionnement cognitif. Psychologie Française, 29, 231-234.

Irwin, G., Hanton, S., \& Kerwin, D.G. (2005). The conceptual process of skill progression development in artistic gymnastics. Journal of Sports Sciences, 23 (10), 10891099.

Jemni, M., Friemel, F., Sands, W., \& Mikesky, A. (2001). Evolution du profil physiologique des gymnastes durant les 40 dernières années. Canadian Journal of Applied Physiology, 26(5), 442- 456.

Johnson-Laird, P.N. (1983). Mental models: Towards a cognitive science of language, inference, and consciousness. Cambridge, MA: Harvard.

Jones, D. F., Housner, L.D. \& Kornspan, A.S. (1997). Interactive decision making and behavior of experienced and inexperienced basketball coaches during practice. Journal of Teaching in Physical Education, 16, 454-468.

Kanéko, A. (trad. Fr, Grossetête, M.T. 1980). Gymnastique olympique. Paris : Vigot.

Kaufmann, J.C. (1997). Le cœur à l'ouvrage. Théorie de l'action ménagère. Paris: Nathan. 


\section{eJRIEPS 39 juillet 2016}

Kirshner, D. \& Whitson, J.A. (1997). Situated cognition: social, semiotic, and psychological perspectives. Mahwah, NJ: Erlbaum.

Koch, K. (1980). Gymnastique à l'école primaire. Paris : Vigot.

Kohler, M., \& Nachon, M. (2002). Rôle et place de la démonstration partielle dans l'enseignement de la gymnastique sportive chez deux populations d'enseignants. eJRIEPS, 1, 36 - 49.

Koren, R. (2011). De la rationalité et/ou de l'irrationalité des polémiqueurs : Certitudes et incertitudes », Semen, 31. En ligne http://semen.revues.org/9061

Lakoff, G., \& Johnson, M. (1980). Metaphors we live by. Chicago: University of Chicago Press.

Le Paven, M. (2008). La relation didactique entraîneur / athlète en lancers. Thèse de doctorat non publiée, Université Rennes 2, Rennes.

Léziart, Y. (1997). Savoirs savants et transpositions didactiques en éducation physique et sportive. STAPS, 42, 59-72.

Maturana, U., \& Varela, F. (1994). L'arbre de la connaissance. Paris : Addison Wesley.

Molino, J. (1998). Expérience et connaissance de la musique à l'âge des neurosciences, In E. Darbellay (Dir.), Le temps et la forme. Pour une épistémologie de la connaissance musicale, (pp. 253-272). Genève : Librairie Droz.

Muybridge, E. (1887). Animal locomotion (Vol 1 - Vol 11). Philadelphia : University of Pennsylvania.

Nouillot, P., \& Natta, F. (2002). Modèle d'organisation segmentaire et incidence sur les contenus d'enseignement. Exemple de l'ATR, in J- F. Robin, \& A. Durny (Dir.), Travaux d'actualité en activités gymniques et acrobatiques, (pp. 103-107), Dossier EPS, 57. Paris : Revue EPS.

Penelaud, O. (2010). Le paradigme de l'énaction aujourd'hui. Apports et limites d'une théorie cognitive « révolutionnaire ». PLASTIR, 18. En ligne http://plasticitessciences-arts.org/PLASTIR/Penelaud\%20P18.pdf

Pepper, S.C. (1942). World hypotheses. Berkeley.

Perrenoud, P., Altet, M., Charlier, E., \& Paquay, L. (1996). Fécondes incertitudes ou comment former des enseignants avant d'avoir toutes les réponses, in L. Paquay, M. Altet, E. Charlier, et P. Perrenoud (Dir.), Former des enseignants professionnels. Quelles stratégies, quelles compétences? (pp. 240-253). Bruxelles : De Boeck Université. 


\section{eJRIEPS 39 juillet 2016}

Perrey, S. (2007). La biomécanique au service de la préparation physique, in G. Millet, \& D. Le Gallain, La préparation physique : optimisation et limites de la performance sportive, (pp. 204-216) ? Paris, Elsevier.

Polanyi, M. (1966). The Tacit Dimension. University of Chicago Press: Chicago.

Pozzo, T., \& Studeny, C. (1987). Théorie et pratique des sports acrobatiques. Paris: Vigot.

Rolland, C. (2011). La connaissance des entraîneurs experts en gymnastique artistique entre perception de formes et intelligibilité pratique. Thèse de doctorat non publiée, Université Clermont-Ferrand II, Clermont-Ferrand.

Rosenthal, V. (1993). Cognition, vie et ... temps. Intellectica, 16, 175-207.

Rosenthal, V., \& Visetti, Y.M. (1999). Sens et temps de la gestalt. Intellectica, 28, 147-227.

Salmela, J. (1982). Fundamental demands of gymnastics. SNIPES Journal, 5, 11-18.

Salmela, J.H., Russel, S.J., Côté, J., \& Baria, A. (1994). The structure of expert knowledge in coaches. In J. Nitsch (Ed.), Advances in Sport Psychology (pp. 56-65). Köln: Bundesinstitut für sportpsychologie.

Schneuwly, B. (2012). Praticien réflexif, réflexion et travail enseignant : l'oubli de l'objet et des outils d'enseignement. In, M. tardif, C. Borges, \& A. Malo, Le virage réflexif en éducation (pp. 73-91). Bruxelles : De Boeck.

Schön, D.A. (1983). The reflective practitioner. How professionals think in action. NewYork: Basic Books.

Shulman, L.S. (1987). Knowledge and teaching. Foundations of the New Reform. Harward Educational Review, 57 (1), 1-22.

Strauss, A. L. (1992). La méthode comparative continue en analyse qualitative. In I. Baszanger (Ed.), La trame de la négociation, sociologie qualitative et interactionnisme, (pp. 283-311). Paris : L'Harmattan.

Steiner, P. (2008). Sciences cognitives, tournant pragmatique et horizons pragmatistes. Tracés, 15, 85-105Suchman, L. (1987). Plans and situated action. Cambridge: Cambridge University Press.

Tanguay, E., \& Tousignant, M. (1998, July). What do coaches look for during a training session? Paper presented at the AIESEP World Sport Science Congress. Adelphi University, New-York.

Tardif, M., Borges C., \& Malo, A. (2012). Le virage réflexif en éducation. Bruxelles : De Boeck. 
eJRIEPS 39 juillet 2016

Varela, F. J. (1989). Autonomie et connaissance. Essai sur le vivant. Paris : Seuil.

Varela, F., Thompson, E., \& Rosch, E. (1993). L'inscription corporelle de l'esprit. Sciences cognitives et expérience humaine. Paris : Seuil.

Vermersch, P. (1990). Questionner l'action : l'entretien d'explicitation, Psychologie française, 35-3, 227-235.

Vermersch, P. (1994). L'entretien d'explicitation. Paris : ESF.

Vosnadiou, S. \& Brewer, W.F. (1992). Mental models of the earth: A study of conceptual change in childhood. Cognitive Psychology, 24, 535-585. 